\title{
Design of Dual-Band Waveguide Transformers
}

\author{
Uwe Rosenberg ${ }^{1}$, Jens Bornemann ${ }^{2}$ and Smain Amari ${ }^{3}$ \\ ${ }^{1}$ Marconi Communications GmbH, D-71520 Backnang, Germany \\ ${ }^{2}$ University of Victoria, Victoria, BC, Canada V8W 3P6 \\ ${ }^{3}$ Royal Military College of Canada, Kingston, ON, Canada K7K 7B4
}

\begin{abstract}
$A B S T R A C T$ - This paper presents guidelines for the design of dual-band transformers in both rectangular and ridge waveguide technologies. Four to eight transformer sections are used to satisfy specifications for advanced satellite and terrestrial communications in $\mathrm{C}$-band and $\mathrm{Ku}$-band. Compact designs are obtained by synthesis of wideband components, which are then optimized for dual-band application. Measurements of a Kuband prototype verify the design approach.
\end{abstract}

Index Terms - Impedance transformers, waveguide transitions, waveguide discontinuities, ridge waveguides, satellite communications.

\section{INTRODUCTION}

The theory and design of transmission-line and waveguide impedance transformers are well documented and understood [1], [2]. Many of the underlying principles found their way into computer-aided synthesis and design algorithms, e.g. [3], but only little work on transformers focused on the dual-band nature used, for example, in antenna feeds of modern communication systems such as satellite and terrestrial lineof-sight applications.

Only recently has this topic been theoretically addressed. Design guidelines for dual-band transmission-line transformers with respect to the lengths and impedance levels of individual transformer sections have been developed [4] [8]. The main items of interest were the design of the upper band as a harmonic of the fundamental band and the reduction of component size by using shorter sections. However, all derivations presented in [4] - [8] dealt exclusively with twosection transformers which by no means satisfy the demanding performance specifications of modern communication systems. Moreover, they are based on TEMlike characteristics and fail to address dispersion effects in waveguide technology.

Therefore, in this paper a number of dual-band waveguide transformer designs are presented, which use four to eight sections. They are relatively compact and display performances adequate for today's design challenges. The initial investigation focuses on C-band as one of the classic dual-band satellite application. Dual-band rectangular waveguide transformers (Fig. 1a) are used for regular C-band coverage while ridge waveguide technology (Fig. 1b) is employed for extended frequency ranges. Finally, a compact prototype for advanced Ku-band satellite applications demonstrates high-performance properties.

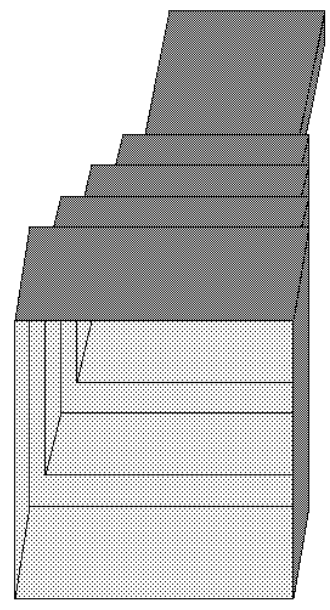

(a)

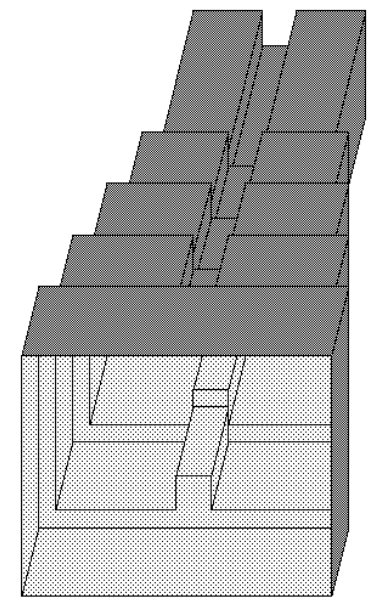

(b)
Fig. 1 Three-section dual-band transformers in rectangular (a) and ridge (b) waveguide technology.

\section{DESIGN CONSIDERATIONS}

Dual-band transformer designs with a relatively wide separation between the bands are based on two different strategies.

First, a regular quarter-wavelength transformer can be designed for the lower band, e.g. according to [3]. Operation in the upper band can then be achieved by utilizing its harmonic performance at three quarter-wavelengths. Such an example is shown in Fig. 2 (dashed line) for a dual-C-band transformer from a square to regular C-band cross section. The specifications require $38 \mathrm{~dB}$ return loss over the frequency bands $3.6-4.2 \mathrm{GHz}$ and $5.8-6.5 \mathrm{GHz}$. The foursection transformer, which is designed for a midband frequency of $3.9 \mathrm{GHz}$ in the lower band, satisfies the specifications. Due to waveguide dispersion, however, the guided wavelength in the lower band is long $\left(\lambda_{\mathrm{g}}=1.9 \lambda_{0}\right)$ and, therefore, the length of the transformer between the two ports is $182 \mathrm{~mm}$ (c.f. Fig. 2).

Secondly, a broadband transformer can be designed for the entire band from 3.6 to $6.5 \mathrm{GHz}$ (center frequency $5.1 \mathrm{GHz}$, bandwidth $56 \%$ ). Such a performance is shown as the solid 
line in Fig. 2. Of course, more sections (six in this case) are required for broadband operation, but the overall transformer length of $127 \mathrm{~mm}$ is shorter than the previous four-section design due to reduced dispersion $\left(\lambda_{\mathrm{g}}=1.3 \lambda_{0}\right)$.

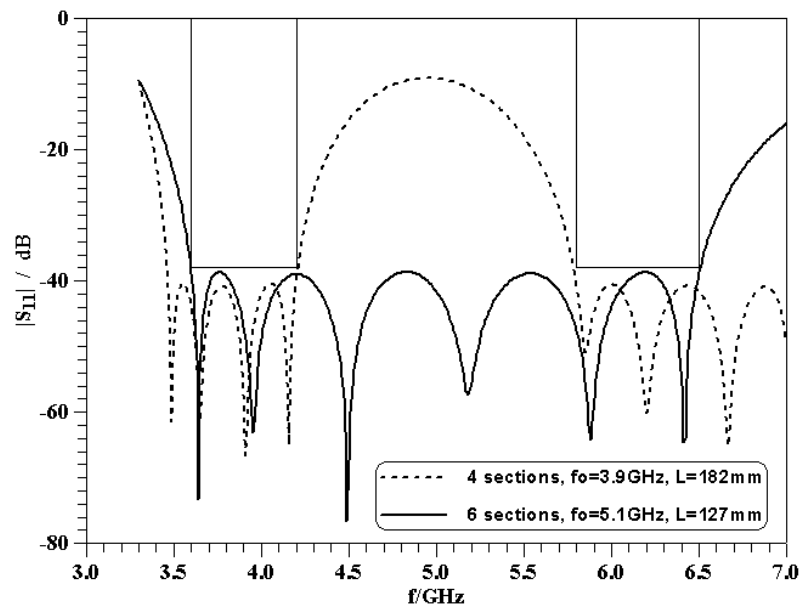

Fig. 2 Performance comparison between two constant-width dualC-band transformers; four-section harmonic and six-section broadband design.

In comparing the performances shown in Fig. 2, it is obvious that the return loss of the six-section design (solid line) within the two bands can be improved if the specifications between the bands are relaxed, i.e., if between the bands, a performance similar to the four-section design (dashed line) is obtained.

Therefore, we recommend the following design strategy:

- Design a broadband transformer covering both bands.

(Note that return-loss specifications are not required to be met in this step.)

- In view of the lack of synthesis procedures for three or more sections, optimize the broadband design under the constraint that the overall length is not increased.

For the results shown in the next section, coupled-integralequations and mode-matching techniques are used as analysis tools. Both techniques have been previously verified by HFSS, e.g. [9], [10]. Optimization is based on a MiniMax routine, e.g. [11].

\section{RESULTS}

Following the above guidelines, the six-section broadband design of Fig. 2 was optimized for return-loss improvement in the two bands. The result is shown in Fig. 3 as a solid dark line. Compared to Fig 2, the in-band return loss is improved by more than $4 \mathrm{~dB}$, and the overall length is slightly reduced by $5 \mathrm{~mm}$.

Also shown in Fig. 2 (gray lines) is a tolerance analysis for maximum deviations of $\pm 0.02 \mathrm{~mm}$ in a fabrication process. Note that the component maintains specifications under such conditions even though this has been verified to be a worstcase scenario (c.f. [12]).

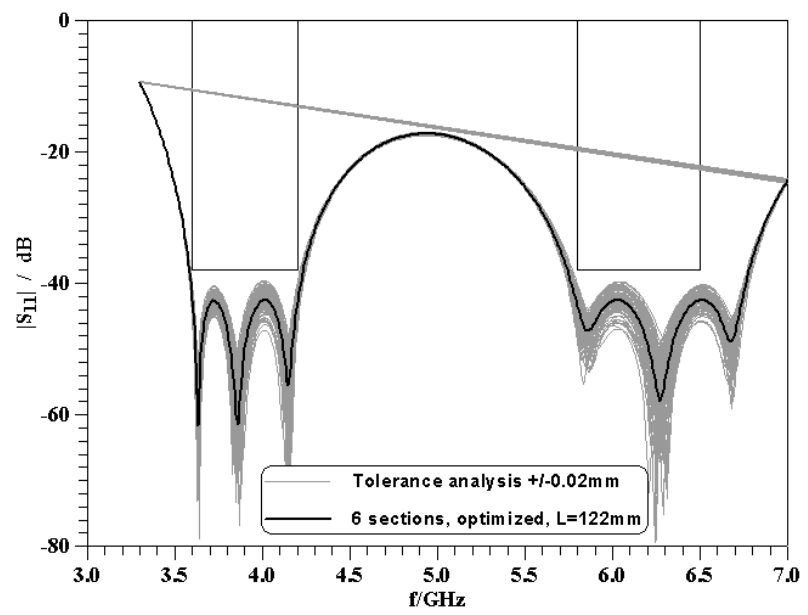

Fig. 3 Performance and tolerance analysis $( \pm 0.02 \mathrm{~mm})$ of a sixsection constant-width dual-band rectangular waveguide transformer in C-band.

For extended C-band operation, e.g. 3.4 - $4.2 \mathrm{GHz}$ and 5.85 $-6.65 \mathrm{GHz}$ (e.g. Satcom INTELSAT), rectangular waveguide transformers are avoided due to the problem of higher order mode excitation and propagation in the waveguide system. In such applications, a square input waveguide interface of an antenna feed may have to be connected to a ridge waveguide (c.f. Fig. 1b) cross section, providing sole fundamental mode propagation. Commonly, these designs have slightly reduced return-loss specifications $(30 \mathrm{~dB})$ compared to the standard rectangular waveguide design.

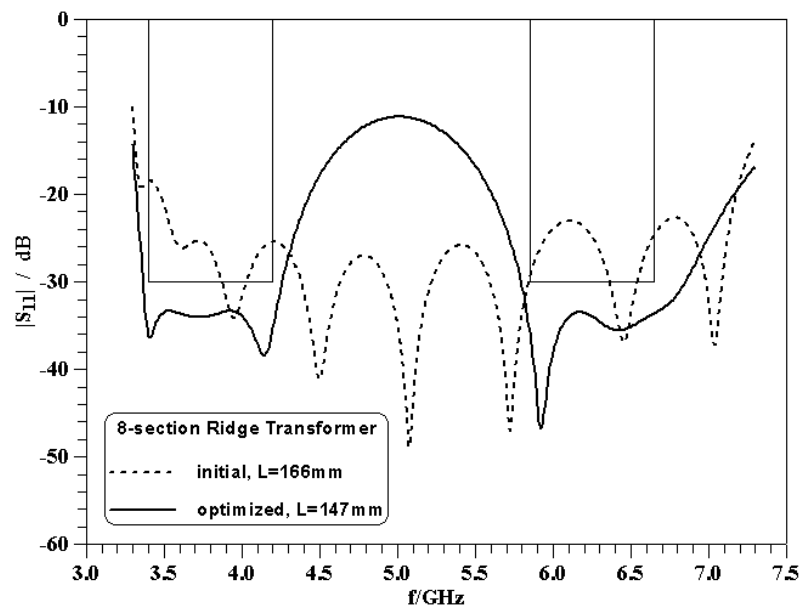

Fig. 4 Initial and optimized performances of a dual-band ridge waveguide transformer in C-band according to Fig. $1 \mathrm{~b}$.

Fig. 4 shows the performance of a dual-band ridge waveguide transformer. According to the design guidelines, an initial broadband design is performed. This is shown for an eight-section transformer as a dashed line in Fig. 4. Note that this design has been obtained by adjusting the gap between 
the ridges to fit a broadband impedance profile and that it does not satisfy the return-loss specifications in either one of the bands. The component's performance after optimization is depicted as a solid line. Not only the design specifications are satisfied in both bands, the transformer length is also reduced by $19 \mathrm{~mm}$, i.e. more than ten percent. (For verification of the code by HFSS, the reader is referred to [10].)

Several $\mathrm{Ku}$-band satellite applications utilize the $11.7-12.5$ $\mathrm{GHz}$ and $17.3-18.1 \mathrm{GHz}$ frequency ranges. The input port is a $16.3 \mathrm{~mm}$ square waveguide and the output port a standard WR62. Therefore, this is not a constant-width design but follows the profile in Fig. 1a. Fig. 5 shows the performance of a four-section dual-band transformer that satisfies the indicated design specifications. The overall length is only 25.5 $\mathrm{mm}$.

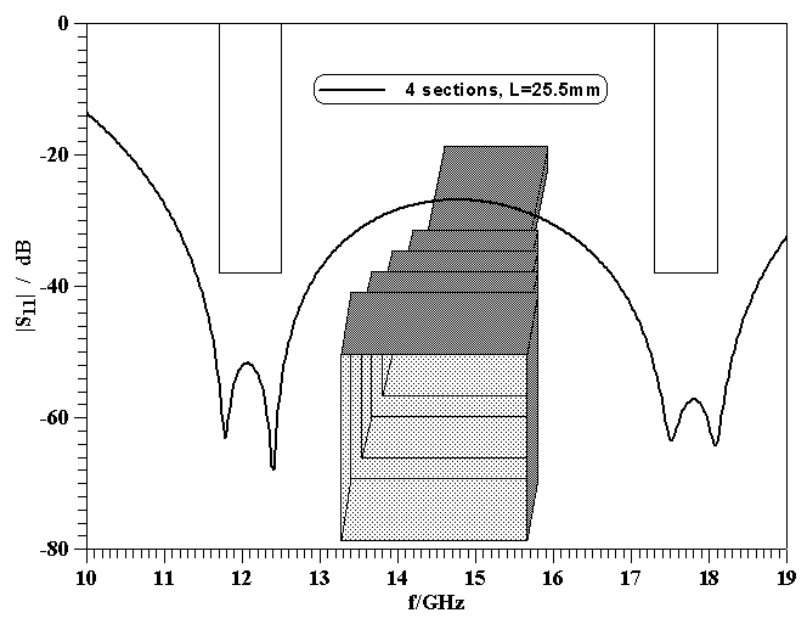

Fig. 5 Four-section dual-band rectangular waveguide transformer for Ku-band applications.

In order to experimentally verify the design approach presented here, the Ku-band transformer in Fig. 5 was reoptimized by the commercial software package $\mu$ Wave Wizard $^{\odot}$ to include an end-mill cutter radius of $3 \mathrm{~mm}$ for ease of fabrication.

Fig. 6 compares the computed (solid line) with the measured (dashed line) performance. Very good agreement is obtained over the entire frequency range, and the specifications of $38 \mathrm{~dB}$ return loss in the two frequency bands are met. Note that the maximum difference between the computed and measured reflection coefficient is less than one percent. The deviations of the curves result mainly from limitations of the achievable accuracy of the test equipment (VNA 8510) for the measurement of reflection coefficient values below $-40 \mathrm{~dB}$. This holds in particular for the lower frequency range from $10 \mathrm{GHz}$ to $12 \mathrm{GHz}$ which is rather close to the cutoff frequencies of the connecting waveguides and consequently below their recommended operating frequency bands (WR62, $\mathrm{f}_{\mathrm{c}}=9.5 \mathrm{GHz}$, recommended band 12.4 - $18 \mathrm{GHz}$; square waveguide $16.3 \mathrm{~mm}, \mathrm{f}_{\mathrm{c}}=9.2 \mathrm{GHz}$ ). Due to strong dispersion, the inherent return loss of the used precision load for the square waveguide port decreases from $46 \mathrm{~dB}$ at 12 $\mathrm{GHz}$ to $34 \mathrm{~dB}$ at $10 \mathrm{GHz}$. However, this is still sufficient for the quality of the measurement, since the band of interest is close to $12 \mathrm{GHz}$ and the return loss of the dual-band transformer decreases rapidly to $14 \mathrm{~dB}$ at $10 \mathrm{GHz}$ (c.f. Fig. 6).

The measured insertion loss is better than $0.05 \mathrm{~dB}$ in both bands. A photograph of the prototype dual-band transformer is depicted in Fig. 7.

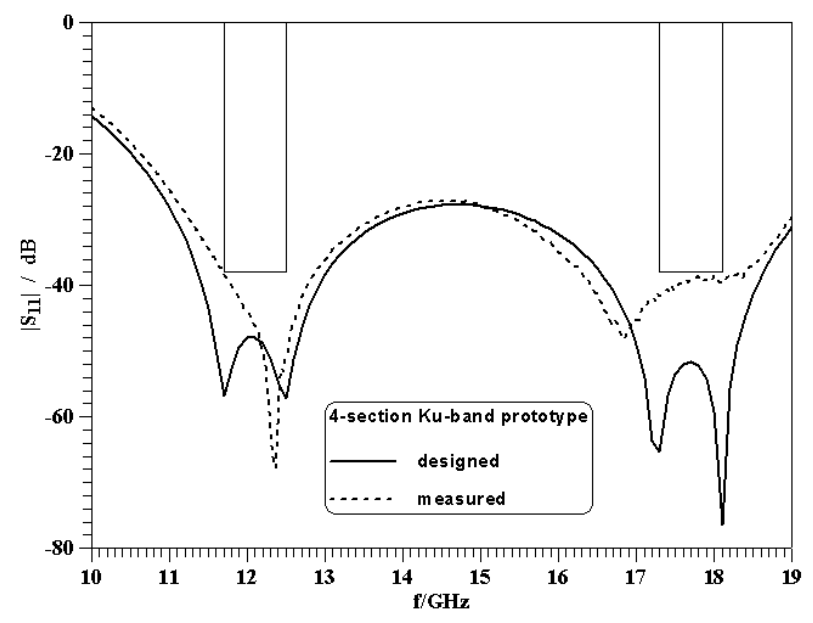

Fig. 6 Comparison between measured and computed results for the four-section dual-band transformer prototype; $\mu$ Wave Wizard ${ }^{\odot}$ (solid line), measurement (dashed line).

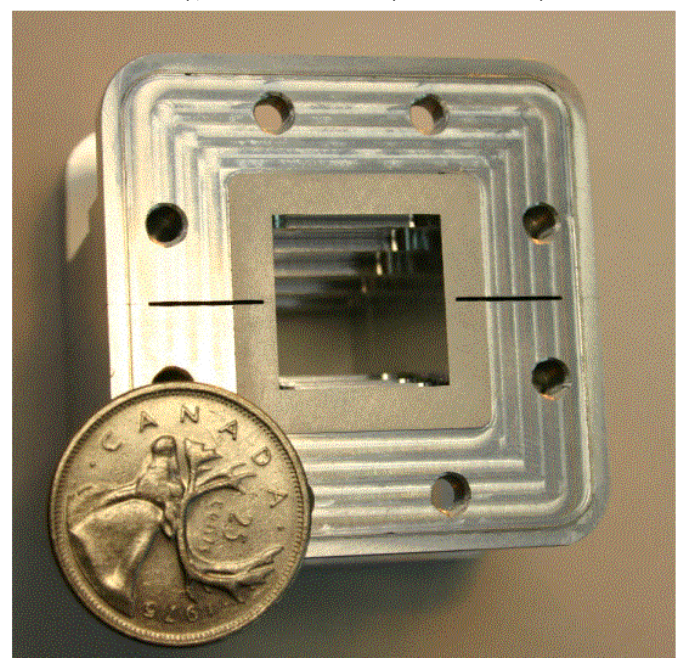

Fig. 7 Photograph of the dual-Ku-band prototype transformer and size comparison with a Canadian quarter coin.

\section{CONCLUSIONS}

The design strategy for dual-band waveguide transformers results in short and compact components that satisfy the stringent specifications of today's satellite and terrestrial applications. Various designs in rectangular and ridge waveguide technologies demonstrate the feasibility of the approach, which is verified through measurements performed at a $\mathrm{Ku}$-band prototype. 
Acknowledgment: The prototype was fabricated at the Herzberg Institute of Astrophysics (HIA) in Victoria, Canada. The authors are greatly indebted to Stéphane Claude, Kei Szeto and their teams at HIA.

\section{REFERENCES}

[1] S.B. Cohn, "Optimum design of stepped transmission-line transformers", IRE Trans. Microwave Theory Tech., Vol. 4, pp. 16-21, Apr. 1955.

[2] G. L. Matthaei, L. Young and E. M. T. Jones, Microwave Filters, Impedance Matching Networks and Coupling Structures, Artech House, Dedham: 1980.

[3] J. Uher, J. Bornemann and U. Rosenberg, Waveguide Components for Antenna Feed Systems: Theory and CAD, Artech House, Norwood: 1993.

[4] Y.L. Chow and K.L. Wan, "A transformer of one-third wavelength in two sections - For a frequency and its first harmonic", Microwave Wireless Components Lett., Vol. 12, pp. 22-23, Jan. 2002.

[5] C. Monzon, "Analytical derivation of a two-section impedance transformer for a frequency and its first harmonic," IEEE Microwave Wireless Comp. Lett., Vol. 12, p. 381-382, Oct. 2002.
[6] C. Monzon, "A small dual-frequency transformer in two sections", IEEE Trans. Microwave Theory Tech., Vol. 51, pp. $1157-1161$, Apr. 2003.

[7] S.J. Orfanidis, "A two-section dual-band Chebyshev impedance transformer", IEEE Microwave Wireless Comp. Lett., Vol. 13, pp. 382 - 384, Sep. 2003.

[8] M.-J. Park and B. Lee, "Design of dual band impedance transformers", in Proc. Asia-Pacific Microwave Conf., pp. 529-532, Seoul, Korea, Nov. 2003.

[9] J. Bornemann, U. Rosenberg, S. Amari and R. Vahldieck, "Edge-conditioned vector basis functions for the analysis and optimization of rectangular waveguide dual-mode filters," in IEEE MTT-S Int. Microwave Symp. Dig., pp. 1695-1698, Anaheim, USA, June 1999.

[10] V.A. Labay and J. Bornemann, "Broadband rectangularto-ridge-to-T-septum waveguide transformers", Microwave Opt. Technol. Lett., Vol. 43, pp. 183-185, Nov. 2004.

[11]K. Madsen, H. Schaer-Jacobsen and J. Voldby, "Automated minimax design of networks", IEEE Trans. Circuits Systems, Vol. 22, pp. 791-796, Oct. 1975.

[12] J. Bornemann, U. Rosenberg, S. Amari and R. Vahldieck, "Tolerance analysis of bypass-, cross- and direct-coupled rectangular waveguide bandpass filters", IEE Proc.Microw. Antennas Propag., Vol. 141, Oct. 2004. 